\title{
DENOSUMAB TREATMENT IN A RARE, NEGLECTED GIANT CELL TUMOR (GCT) OF THE FEMORAL NECK: CASE REPORT AND LITERATURE REVIEW
}

doi: 10.2478/rojost-2018-0074

\author{
R. Marinescü, D. Lăptoiu 2, I. Botezatu 2, S. Ciumeica ${ }^{2}$, A. Bunea ${ }^{2}$, G. Ștefan ${ }^{2}$ \\ "Carol Davila" University of Medicine and Pharmacy, Bucharest, Romania \\ ${ }^{2}$ Colentina Clinical Hospital, Bucharest, Romania
}

Introduction. GCT resembles an aggressive benign tumor of bone and its evolution based on the histological features is unpredictable. About $50 \%$ of the cases are located around the knee (proximal femur and distal tibia), with the proximal humerus and distal radius representing the third and fourth most common sites. Femoral neck location is unusual. We report a case of GCT located at the femoral neck level, in a 19-year-old female.

Case presentation. Onset was hidden by pathologic femoral neck fracture and, due to insidious symptoms, proper diagnosis and treatment were neglected for almost six months. After 6 months, the case was referred to our clinic and re-evaluated with complete examination and biopsy. Wide resection and tumoral arthroplasty of the hip was performed. Postoperative complete recovery was achieved and the patient returned to previous activities. After 2 years of normal clinical evolution, a neurological severe issue appeared; at this time, a cerebral metastasis was diagnosed. Once the positive diagnosis was achieved, $120 \mathrm{mg}$ Denosumab treatment was initiated monthly. At 16 months follow-up, the patient was symptom free and continued Denosumab treatment.

Discussion. Denosumab is a human monoclonal IGG2 antibody inhibiting osteoclast differentiation, activation, and survival with applicable suppression of bone turnover in patients with multiple myeloma, osteolytic bone disease, and bone metastases from breast and prostate cancer. It is also a useful drug for managing the GCT of bone and one excellent option in metastatic GCT. The long time safety and complications, especially in young female patients, are to be proven. Conclusions. Early diagnosis and accurate management of GCT are mandatory in order to achieve good long-term clinical results. Denosumab treatment may be necessary in order to avoid secondary metastasis or local recurrence.

Keywords: giant cell tumor, femoral neck, cerebral metastasis, denosumab, surgery 